\title{
Amide proton transfer imaging in differentiation of type II and type I endometrial carcinoma: a pilot study
}

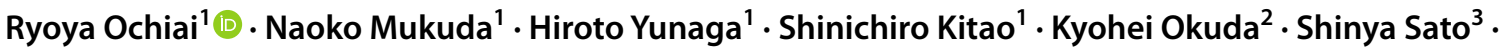 \\ Tetsuro Oishi ${ }^{3} \cdot$ Mitsuharu Miyoshi $^{4} \cdot$ Atsushi Nozaki $^{4} \cdot$ Shinya Fujii ${ }^{1}$
}

Received: 7 April 2021 / Accepted: 6 September 2021 / Published online: 15 September 2021

(c) The Author(s) 2021

\begin{abstract}
Purpose This study aimed at evaluating the efficacy of amide proton transfer (APT) imaging in differentiation of type II and type I uterine endometrial carcinoma.

Materials and methods Thirty-three patients diagnosed with uterine endometrial carcinoma, including 24 with type I and 9 with type II carcinomas, underwent APT imaging. Two readers evaluated the magnetization transfer ratio at $3.5 \mathrm{ppm}$ $\left[\mathrm{MTR}_{\text {asym }}(3.5 \mathrm{ppm})\right]$ in each type of carcinoma. The average $\mathrm{MTR}_{\text {asym }}\left(\mathrm{APT}_{\text {mean }}\right)$ and the maximum $\mathrm{MTR}_{\text {asym }}\left(\mathrm{APT}_{\max }\right)$ were analyzed. The receiver operating characteristic (ROC) curve analysis was performed.

Results The $\mathrm{APT}_{\max }$ was significantly higher in type II carcinomas than in type I carcinomas (reader1, $p=0.004$; reader 2, $p=0.014$; respectively). However, $\mathrm{APT}_{\text {mean }}$ showed no significant difference between type I and II carcinomas. Based on the results reported by reader 1, the area under the curve (AUC) pertaining to the $\mathrm{APT}_{\max }$ for distinguishing type I from type II carcinomas was 0.826 , with a cut-off, sensitivity, and specificity of $9.90 \%, 66.7 \%$, and $91.3 \%$, respectively. Moreover, based on the results reported by reader 2 , the AUC was 0.750 , with a cut-off, sensitivity, and specificity of $9.80 \%, 62.5 \%$, and $87.5 \%$, respectively.
\end{abstract}

Conclusion APT imaging has the potential to determine the type of endometrial cancer.

Keywords Chemical exchange saturation transfer $\cdot$ Magnetic resonance imaging $\cdot$ Uterine $\cdot$ Differentiation $\cdot$ Histological

\section{Introduction}

Endometrial cancer is the most common malignancy of the female reproductive organs in developed countries, including the United States and Europe. The prevalence of the disease increases with the age of the population and the

Ryoya Ochiai

r.ochiaifff@gmail.com

1 Division of Radiology, Department of Multidisciplinary Internal Medicine, Faculty of Medicine, Tottori University, 36-1, Nishi-cho, Yonago, Tottori 683-8504, Japan

2 Department of Clinical Radiology, Tottori University Hospital, Yonago, Tottori 683-8504, Japan

3 Division of Reproductive-Perinatal Medicine and Gynecologic Oncology, Department of Obstetrics and Gynecology, Faculty of Medicine, Tottori University, Yonago, Tottori 683-8504, Japan

4 MR Applications \& Workflow, GE Healthcare, 4-7-127 Asahigaoka Hino-shi, Tokyo 191-8503, Japan overall increase in the prevalence of obesity and metabolic syndromes in developed countries [1-4]. The prognosis of endometrial cancer is influenced by several factors, including the International Federation of Gynecology and Obstetrics (FIGO) staging system, vascular invasion, adnexal involvement, and lymph node metastasis [5, 6]. Most endometrial cancers are confined to the corpus uterus and have a good prognosis. However, the prognosis of these patients varies greatly, depending on three major factors: histopathological type, histological grade, and depth of myometrial invasion [5].

Bokhman [7] revealed that endometrial cancer is categorized into two types: type I and type II. Type I carcinoma is associated with estrogen hyperplasia, endometrial hyperplasia, frequent expression of estrogen and progesterone receptors, and younger age, whereas type II carcinoma, which is unrelated to estrogen, is associated with endometrial atrophy and older age [7-12]. Type I carcinoma, comprising well or moderately differentiated endometrial carcinoma, accounts for $80-90 \%$ of all 
endometrial carcinomas. Type II carcinoma, comprising histological types with a strong tendency to invade the myometrium, such as serous carcinoma and clear cell carcinoma, accounts for the remaining $10-20 \%$. The classification of poorly differentiated endometrioid carcinoma as either type varies from report to report [8-12]. Although new classification methods based on the Cancer Genome Atlas (TCGA) currently have been investigated [8], the classification of endometrial cancer into type I and type II is clinically important to determine the treatment strategy. In fact, the National Comprehensive Cancer Network 2020 Guidelines [13] revealed that the combined use of pelvic and aortic lymphadenectomy may be considered in the management of type II carcinoma, owing to the high frequency of lymph node metastases. The guidelines also state that fertility preservation is not recommended, even though the cancer is confined to the uterus in type II carcinoma cases. Consequently, the preoperative differentiation of type II from type I carcinoma is very important to formulate a surgical treatment plan.

Amide proton transfer (APT) imaging is a novel imaging technique that uses endogenous contrast by exchange saturation transfer (CEST) to detect amide protons $(-\mathrm{NH})$ in lowconcentration solutes, such as mobile proteins and peptides in tissues or tumors [14]. Mobile proteins and peptides are thought to have a close relationship with tumor growth activity. The clinical utility of APT imaging has already been demonstrated in glioma, lung cancer, prostate cancer, and rectal cancers [15-18]. In gynecology, APT signals have demonstrated a correlation with the histologic grades of endometrioid endometrial carcinoma [19] and the efficacy in differentiating cervical cancer from normal cervical tissue [20].

The purpose of the current study was to characterize type II endometrial carcinoma using APT imaging and to evaluate the diagnostic accuracy of APT imaging in differentiating type II from type I carcinoma.

\section{Materials and methods}

\section{Patients}

The present study was approved by the Ethics Committee of our hospital, and all patients provided signed informed consent prior to scanning.

Between December 2017 and August 2020, 63 consecutive female patients underwent magnetic resonance imaging (MRI) because of the suspicion of endometrial cancer (Fig. 1). Among these patients, 30 were excluded for the following reasons: (1) surgery was not performed $(n=1)$; (2) lesions were too small to be recognized on MRI $(n=3)$; (3) absence of endometrial cancer ( $n=7$, two endometrial polyps, one atypical endometrial hyperplasia, one carcinosarcoma, one endometrial stromal sarcoma, and two patients with no significant tumor); (4) tumors with a mixture of two histological types $(n=5)$; (5) incomplete scanning sequences or the presence of too many motion/metal/ air imaging artifacts $(n=8)$; (6) the selected APT image section did not show the maximum tumor area $(n=4)$; (7) patients underwent surgery after 100 days following MRI on account of neoadjuvant chemotherapy $(n=2)$. The

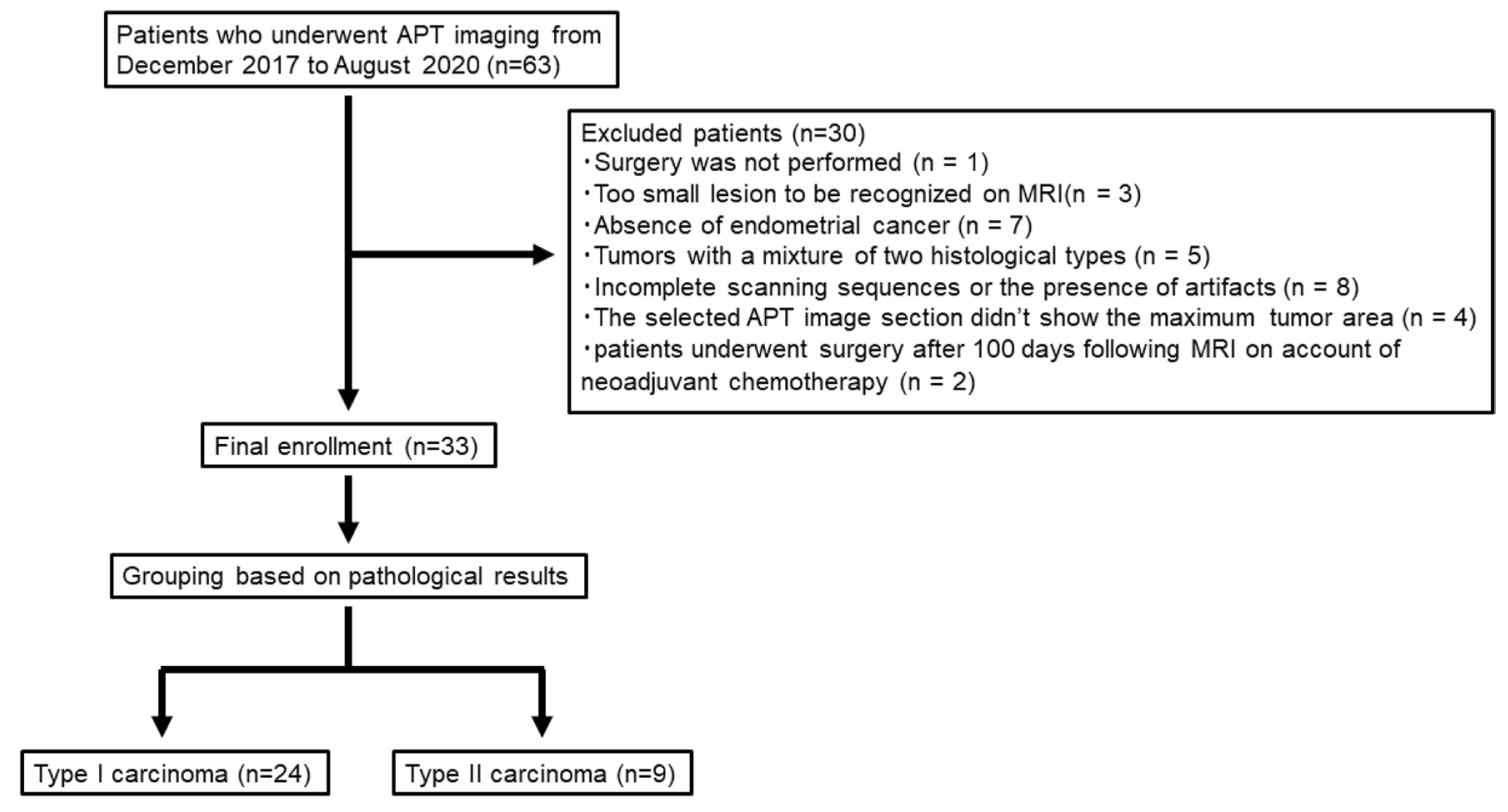

Fig. 1 Flowchart depicting the patient selection 
final study population consisted of 33 patients (mean age 57.8 years; age range $37-72$ years) with newly diagnosed endometrial carcinoma. All patients underwent surgery within 8-77 days after MR examination (mean time interval: 38 days). Macroscopic and microscopic pathological specimens were obtained during surgery.

On the basis of histological features, the well-differentiated (grade 1) and moderately (grade 2) differentiated endometrioid endometrial carcinomas were categorized as type I and poorly differentiated (grade 3 ) endometrioid endometrial carcinomas, serous carcinomas, and clear cell carcinomas were categorized as type II.

\section{MRI technique}

MRI was performed using a 3 T MR system (Discovery MR750, GE Medical Systems, Milwaukee, Wisconsin, USA). A geometry embracing method anterior array 18 channel receiver array coil was used for signal reception. Before the examination, the patients were required to have full bladders. Conventional MRI parameters were as follows: sagittal T1-weighted imaging (T1WI) without fat suppression (field of view [FOV]: $25 \times 25 \mathrm{~cm}^{2}$; slice thickness: $2.0 \mathrm{~mm}$; spacing: $2.0 \mathrm{~mm}$; number of slices: 82; repetition time [TR]: $4.6 \mathrm{~ms}$; echo time [TE]: $2.2 \mathrm{~ms}$; matrix: $512 \times 512$ ), sagittal T2-weighted imaging (T2WI) (field of view [FOV]: $25 \times 25 \mathrm{~cm}^{2}$; slice thickness: $3.0 \mathrm{~mm}$; spacing: $3.6 \mathrm{~mm}$; number of slices: 31 ; repetition time [TR]: $6900 \mathrm{~ms}$; echo time [TE]: $82 \mathrm{~ms}$; matrix: $512 \times 512$ ), sagittal diffusion-weighted imaging (DWI) (field of view [FOV]: $24 \times 24 \mathrm{~cm}^{2}$; slice thickness: $3.0 \mathrm{~mm}$; spacing: $4.2 \mathrm{~mm}$; number of slices: 23; repetition time [TR]: $4800 \mathrm{~ms}$; echo time [TE]: $68 \mathrm{~ms}$; matrix: $256 \times 256$; number of excitations: $6 ; b$ values: 0 and 800 ). After these sequences were acquired, $0.2 \mathrm{~mL} / \mathrm{kg}$ of contrast agents (meglumine gadoterate or gadoteridol) were administered intravenously at a rate of $2.5 \mathrm{~mL} / \mathrm{s}$, followed by $30 \mathrm{~mL}$ of saline flush.

Two-dimensional sagittal APT imaging was performed using single-shot fast-spin echo (SSFSE) acquisition before the administration of contrast agents. The scanning parameters were as follows: TR: $5724 \mathrm{~ms}$; echo time (TE): $31 \mathrm{~ms}$; FOV: $30 \times 24 \mathrm{~cm}^{2}$; matrix: $128 \times 128$; layer thickness: $5.0 \mathrm{~mm}$; saturation pulse (RF): $1.7 \mu \mathrm{T}$; RF type: phase cycle; saturation time: $2500 \mathrm{~ms}$; total acquisition time: $246 \mathrm{~s}$. We used 43 images for these sequences, which comprised first idling, second image for S0 without saturation, third idling, and B0 correction images using water saturation shift referencing $( \pm 240, \pm 192, \pm 144, \pm 96, \pm 48,0 \mathrm{~Hz})$, and $\mathrm{NH}$ images $( \pm 896, \pm 832, \pm 768, \pm 704, \pm 640, \pm 576, \pm 512$ $\pm 448, \pm 384, \pm 320, \pm 256, \pm 192, \pm 128, \pm 64,0 \mathrm{~Hz})$. The $\operatorname{MTR}_{\text {asym }}(3.5 \mathrm{ppm})$ was calculated at $3.5 \mathrm{ppm}$.

\section{Image analysis}

The APT images were acquired through a single section selected by the radiological technicians as the tumor showing the maximum tumor area on sagittal T2WI and DWI. Subsequently, the MR data were analyzed using a clinical viewer (EV Insite; PSP, Tokyo, Japan). Images were independently analyzed by two radiologists (R.O. and N.M., with 3 and 8 years of experience in gynecological MRI, respectively) who were blinded to the histologic findings.

In each case, the radiologists drew a freehand region of interest (ROI) based on the lesion contour on the S0 image, which was obtained at the same scanning as that performed to capture the APT image. Each ROI was drawn by carefully reviewing the T2WI, DWI and contrast-enhanced images to determine the solid part of each tumor. Subsequently, the ROI was copied and pasted onto the $\mathrm{MTR}_{\text {asym }}$ image and apparent diffusion coefficient (ADC) map. We further adjusted the ROI after confirming whether the location of the ROI was appropriate. Tumor MTR $_{\text {asym }}(3.5 \mathrm{ppm})$ and ADC values were determined. The ROI was placed to cover as much of the solid part of the tumor as possible and avoid large vessels, hemorrhage, calcification, cyst, necrosis, and normal myometrium. The average $\mathrm{MTR}_{\text {asym }}$ $\left(\mathrm{APT}_{\text {mean }}\right)$, maximum $\mathrm{MTR}_{\text {asym }}\left(\mathrm{APT}_{\max }\right)$, average $\mathrm{ADC}$ value $\left(\mathrm{ADC}_{\text {mean }}\right)$, and minimum $\mathrm{ADC}$ value $\left(\mathrm{ADC}_{\text {min }}\right)$ of each tumor were evaluated. The MTR $_{\text {asym }}(3.5 \mathrm{ppm})$ can be calculated using the following equation [15]: $\mathrm{MTR}_{\text {asym }}(3.5$ $\mathrm{ppm})=\left(\mathrm{S}_{-3.5 \mathrm{ppm}}-\mathrm{S}_{3.5 \mathrm{ppm}}\right) / \mathrm{S}_{0}$; where $\mathrm{S}_{-3.5 \mathrm{ppm}}$ and $\mathrm{S}_{3.5 \mathrm{ppm}}$ are the signal intensities at -3.5 and $3.5 \mathrm{ppm}$, respectively, and $\mathrm{S}_{0}$ is the signal intensity without saturation.

Additionally, reader 1 evaluated the distribution of the APT signal in the lesion, which was classified as either homogenous or heterogeneous. Heterogeneous distribution was further classified into three types based on the location of the high-signal APT spot-intratumoral focal high signal, high signal by necrosis, and high signal within myometrial invasion near tumor border. We defined high signal by necrosis as cases in which a high-signal APT spot was found around the non-enhancement area of the lesion. Therefore, the distribution of the APT signal was classified into four types.

\section{Statistical analysis}

All statistical analyses were performed using SPSS 25.0. Values of $p<0.05$ were considered to be significant. The Shapiro-Wilk test was used to evaluate the normality of distribution of data, which did not show a normal distribution. Subsequently, the Mann-Whitney $U$ test was used to evaluate the differences between type I and type II carcinomas with regard to the $\mathrm{APT}_{\text {mean }}$ and $\mathrm{APT}_{\max }$. Additionally, we evaluated the differences with regard to the $\mathrm{ADC}_{\text {mean }}$ and 
Table 1 Patient characteristics

\begin{tabular}{llll}
\hline & Type I & Type II & $p$ value \\
\hline Mean age & $56.1 \pm 12.1$ & $61.1 \pm 4.28$ & 0.090 \\
Tumor size (mm) & $49.7 \pm 26.3$ & $73.6 \pm 32.8$ & 0.037 \\
Histological type & & & \\
Endometrioid & & & \\
$\quad$ Grade 1 & 19 & & \\
$\quad$ Grade 2 & 5 & 4 & \\
$\quad$ Grade 3 & & 3 & \\
Serous & & 2 & \\
Clear cell & & & \\
FIGO staging & & 3 & \\
IA & 17 & 2 & \\
IB & 5 & & \\
II & 1 & 3 & \\
IIIA & 1 & & \\
IIIC2 & & & \\
IVB & & &
\end{tabular}

$\mathrm{ADC}_{\text {min }}$. We also evaluated the correlation between $\mathrm{APT}_{\text {mean }}$ and $\mathrm{ADC}_{\text {mean }}$ as well as $\mathrm{APT}_{\text {max }}$ and $\mathrm{ADC}_{\text {min }}$ using Spearman's rank correlation coefficient.

The interobserver agreement for the ROI measurements pertaining to the two readers was analyzed by estimating the interclass correlation coefficient (ICC 0.00-0.20 slight, $0.21-0.40$ fair, $0.41-0.60$ moderate, $0.61-0.80$ substantial, and 0.81-1.00 almost perfect correlation).

ROC curve analysis was used to evaluate the diagnostic accuracy of $\mathrm{APT}_{\text {mean }}$ and $\mathrm{APT}_{\text {max }}$. Additionally, by maximizing the Youden index (defined as sensitivity + specificity - 1), the optimal thresholds and corresponding sensitivities and specificities for the differentiation of type II and type I carcinomas were determined.

\section{Results}

\section{Patient characteristics}

Histopathological evaluation of the 33 postoperative specimens revealed 24 type I and nine type II endometrial carcinomas. Among the 24 type I endometrial carcinomas, 19 were grade 1 , and five were grade 2 endometrioid carcinomas. Among the nine type II endometrial carcinomas, four were grade 3 endometrioid carcinomas, three were serous carcinomas, and two were clear cell carcinomas. The patient characteristics are summarized in Table 1.

\section{APT signals and ADC values in type I and II endometrial carcinoma}

The ICCs pertaining to $\mathrm{APT}_{\text {mean }}$ and $\mathrm{APT}_{\max }$ were 0.86 and 0.90 , respectively, indicating an almost perfect correlation. The ROI area was $7.92 \pm 7.47 \mathrm{~cm}^{2}$ for reader 1 and $8.28 \pm 9.37 \mathrm{~cm}^{2}$ for reader 2 . A summary of the results of the two readers is shown in Tables 2 and 3. The APT max $_{\text {was }}$ significantly higher in type II carcinomas than in type I carcinomas (reader 1: $p=0.004$; reader 2: $p=0.014$ ) (Fig. 2). Conversely, there was no significant difference between type I and type II carcinomas with regard to $\mathrm{APT}_{\text {mean }}$ (reader
Table 2 Comparison between type I and type II carcinomas with regard to the APT and ADC

\begin{tabular}{|c|c|c|c|c|c|c|}
\hline & \multicolumn{3}{|l|}{ Reader 1} & \multicolumn{3}{|l|}{ Reader 2} \\
\hline & Type I & Type II & $p$ & Type I & Type II & $p$ \\
\hline $\mathrm{APT}_{\text {mean }}$ & $2.21 \pm 0.93$ & $2.18 \pm 1.47$ & 0.648 & $2.26 \pm 0.98$ & $1.96 \pm 0.89$ & 0.619 \\
\hline $\mathrm{APT}_{\text {max }}$ & $6.64 \pm 2.46$ & $10.61 \pm 3.23$ & 0.004 & $7.05 \pm 2.37$ & $11.00 \pm 3.69$ & 0.014 \\
\hline $\mathrm{ADC}_{\text {mean }}$ & $1.04 \pm 0.20$ & $1.06 \pm 0.18$ & 0.512 & $1.00 \pm 0.22$ & $1.03 \pm 0.19$ & 0.858 \\
\hline $\mathrm{ADC}_{\min }$ & $0.60 \pm 0.17$ & $0.48 \pm 0.19$ & 0.183 & $0.62 \pm 0.15$ & $0.52 \pm 0.18$ & 0.246 \\
\hline
\end{tabular}

$A P T$ amide proton transfer, $A D C$ apparent diffusion coefficient

\begin{tabular}{|c|c|c|c|c|c|}
\hline & \multicolumn{5}{|c|}{ Endometrioid } \\
\hline & Grade 1 & Grade 2 & Grade 3 & Serous & Clear cell \\
\hline \multicolumn{6}{|l|}{ Reader 1} \\
\hline $\mathrm{APT}_{\text {mean }}$ & $2.32 \pm 1.01$ & $1.81 \pm 0.47$ & $2.35 \pm 2.33$ & $1.97 \pm 0.40$ & $2.19 \pm 0.72$ \\
\hline $\mathrm{APT}_{\text {max }}$ & $6.68 \pm 2.62$ & $6.50 \pm 2.06$ & $10.1 \pm 2.29$ & $12.1 \pm 5.20$ & $9.40 \pm 1.84$ \\
\hline \multicolumn{6}{|l|}{ Reader 2} \\
\hline $\mathrm{APT}_{\text {mean }}$ & $2.38 \pm 1.07$ & $1.83 \pm 0.39$ & $1.78 \pm 1.30$ & $2.16 \pm 0.60$ & $2.04 \pm 0.61$ \\
\hline $\mathrm{APT}_{\text {max }}$ & $7.01 \pm 2.58$ & $7.20 \pm 1.72$ & $10.1 \pm 2.26$ & $13.0 \pm 5.90$ & $9.80 \pm 2.40$ \\
\hline
\end{tabular}

$A P T$ amide proton transfer
Table $3 \mathrm{APT}_{\text {mean }}$ and $\mathrm{APT}_{\text {max }}$ for each histopathological classification 
$\mathbf{a}$

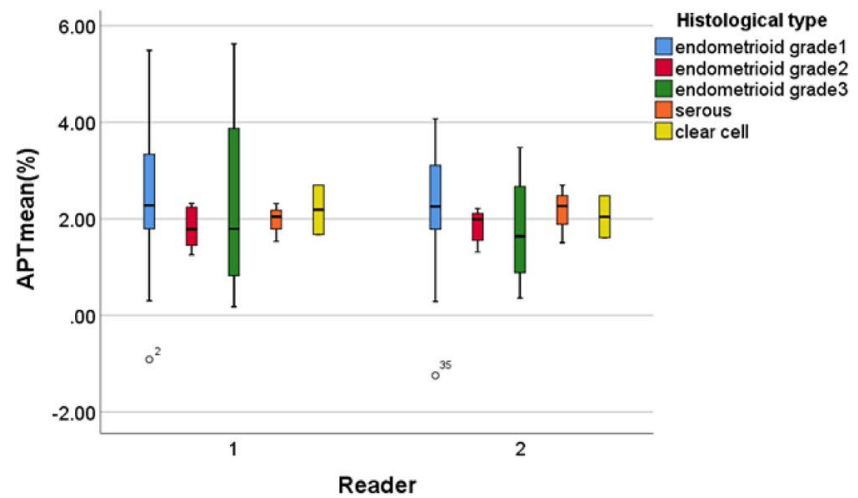

Fig. 2 Box-and-whisker plots show the distribution of the APT (a) and the $\mathrm{APT}_{\max }$ (b) in each histopathological classification of carcinomas. Boxes represent values from the lower to upper quartiles.

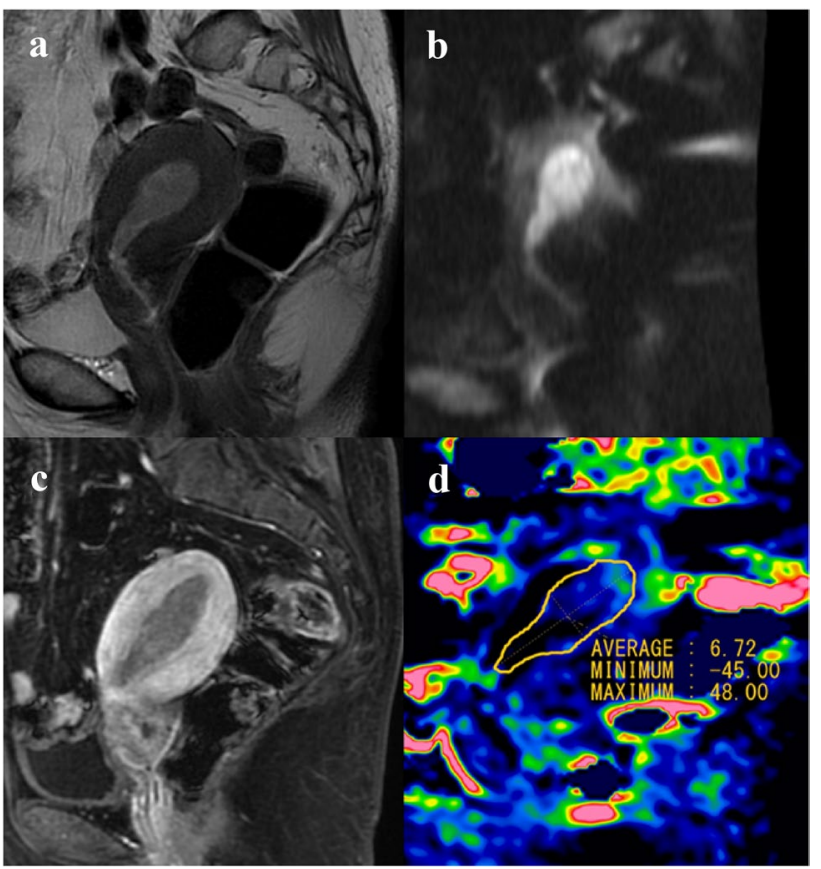

Fig. 3 Images and placement of the ROI in a case of grade 1 endometrioid endometrial carcinoma. a Represents the T2WI, b represents the DWI $(b=800)$, and $\mathbf{c}$ represents the contrast enhanced T1WI, d represents MTR $_{\text {asym }}(3.5 \mathrm{ppm})$ image. The values shown in the figure are multiplied by 1000 to obtain an integer value. Consequently, a value of 10 in the figure is $1.0 \%$. The $\operatorname{MTR}_{\text {asym }}(3.5 \mathrm{ppm})$ image does not show a very high signal area within the ROI. The $\mathrm{APT}_{\text {mean }}$ and $\mathrm{APT}_{\max }$ obtained by reader 1 were $1.89 \%$ and $4.90 \%$, respectively. The $\mathrm{APT}_{\text {mean }}$ and $\mathrm{APT}_{\max }$ obtained by reader 2 were $0.67 \%$ and $4.80 \%$, respectively. The APT signal pattern was classified as homogenous

1: $p=0.648$; reader 2: $p=0.619$ ) (Fig. 2). Figures 3 and 4 show representative images. The APT signal patterns for each histopathological classification are shown in Table 4. b

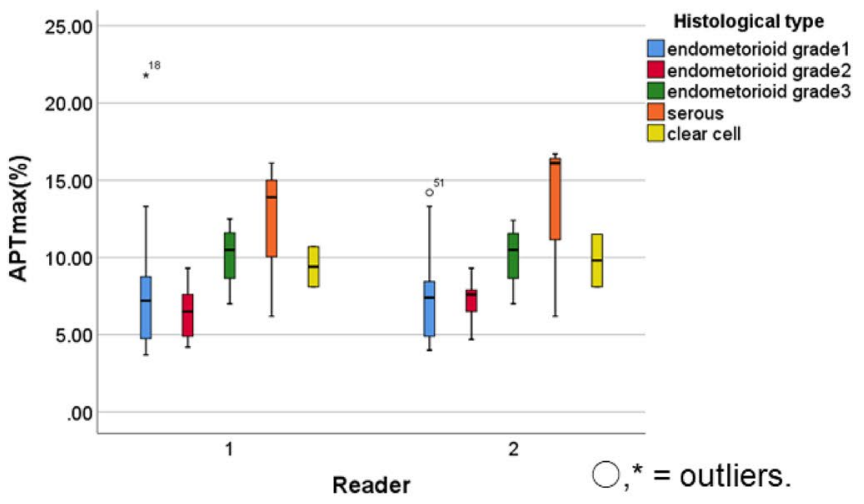

The central line represents the median, and small circles and asterisks represent the extreme values (outliers). Whiskers extend from the minimum to maximum values, excluding the outliers

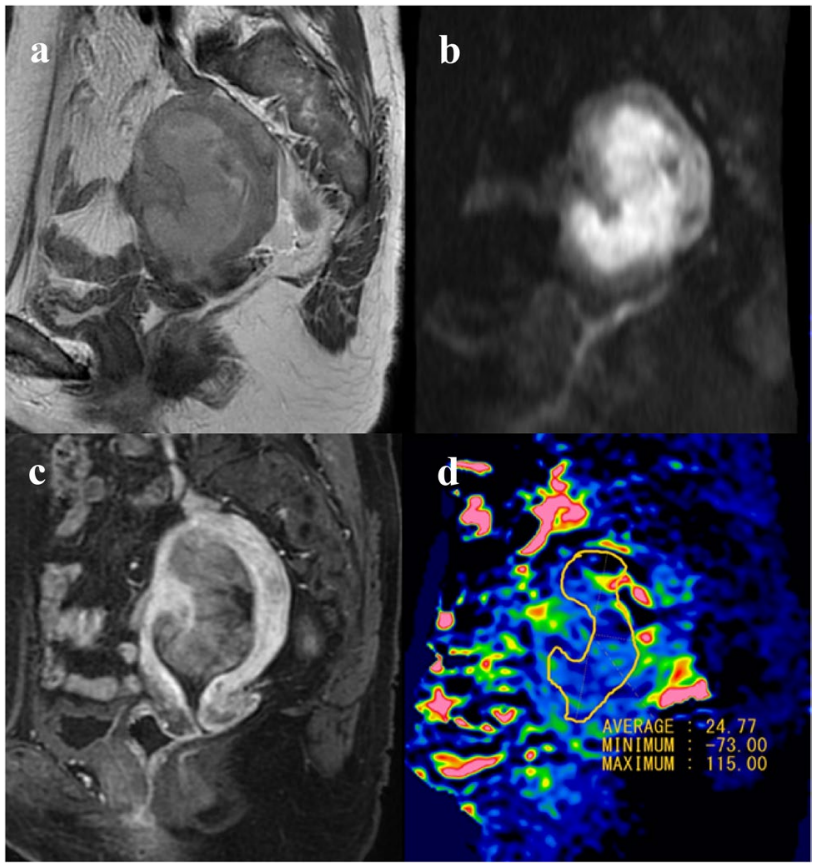

Fig. 4 Images and placement of the ROI in a case of clear cell carcinoma. a Represents the T2WI, b represents the DWI $(b=800)$, and $\mathbf{c}$ represents the contrast enhanced T1WI, $\mathbf{d}$ represents $\mathrm{MTR}_{\text {asym }}$ (3.5 ppm) image. The values shown in the figure are multiplied by 1000 to obtain an integer value. Consequently, a value of 10 in the figure is $1.0 \%$. The MTR asym $_{\text {( }}(3.5 \mathrm{ppm})$ image shows very high signal areas within the ROI. The $\mathrm{APT}_{\text {mean }}$ and $\mathrm{APT}_{\max }$ obtained by reader 1 were $2.78 \%$ and $12.7 \%$, respectively. The $\mathrm{APT}_{\text {mean }}$ and $\mathrm{APT}_{\max }$ obtained by reader 2 were $2.48 \%$ and $11.50 \%$, respectively. The APT signal pattern was classified as high signal by necrosis

We further performed a sub-analysis of only endometrioid carcinomas. The $\mathrm{APT}_{\max }$ was significantly higher in type II (grade 3) endometrioid carcinomas than in type I (grade 1 and 2) endometrioid carcinomas with regard to the 
Table 4 APT signal patterns for each histopathological classification

\begin{tabular}{|c|c|c|c|c|c|}
\hline & \multicolumn{5}{|c|}{ Endometrioid } \\
\hline & Grade 1 & Grade 2 & Grade 3 & Serous & Clear cell \\
\hline Homogenous & 9 & 1 & & & \\
\hline \multicolumn{6}{|l|}{ Heterogenous } \\
\hline Intratumoral focal high signal & 4 & 1 & & 1 & \\
\hline High signal by necrosis & 2 & 2 & 2 & 1 & 2 \\
\hline $\begin{array}{l}\text { High signal within myometrial } \\
\text { invasion near tumor border }\end{array}$ & 4 & 1 & 2 & 1 & \\
\hline
\end{tabular}

results of reader 1 (reader 1: $p=0.034$; reader 2: $p=0.070$ ). There was no significant difference between type I and type II endometrioid carcinomas with regard to $\mathrm{APT}_{\text {mean }}$ (reader $1: p=0.728$; reader $2: p=0.336$ ).

$\mathrm{ADC}_{\text {mean }}$ and $\mathrm{ADC}_{\text {min }}$ were not significantly different between type I and type II carcinomas $\left(\mathrm{ADC}_{\text {mean }}\right.$; reader 1, $p=0.512$; reader $2, p=0.858 ; \mathrm{ADC}_{\min }$, reader $1: p=0.183$; reader 2: $p=0.246$ ).

\section{Correlation between APT signals and ADC values}

We found no significant correlation between $\mathrm{APT}_{\text {mean }}$ and $\mathrm{ADC}_{\text {mean }}$ (reader 1: $p=0.200$, reader 2: $p=0.610$ ) or between $\mathrm{APT}_{\max }$ and $\mathrm{ADC}_{\text {min }}$ (reader 1: $p=0.258$; reader 2: $p=0.974)$.

\section{ROC analysis}

On the basis of the results reported by reader 1, the AUC pertaining to the $\mathrm{APT}_{\max }$ for distinguishing type I from type II carcinomas was 0.826 (95\% confidence interval [CI] $0.667-0.985$ ), with a cut-off, sensitivity, and specificity of $9.90 \%, 66.7 \%$, and $91.3 \%$, respectively. Moreover, on the basis of the results reported by reader 2, the AUC pertaining to the $\mathrm{APT}_{\max }$ for distinguishing type I from type II carcinomas was 0.750 (95\% CI 0.556-0.944), with a cutoff, sensitivity, and specificity of $9.80 \%, 62.5 \%$, and $87.5 \%$, respectively (Fig. 5).

\section{Discussion}

The present study demonstrated that type II endometrial carcinoma has a higher $\mathrm{APT}_{\max }$ than type I endometrial carcinoma, although there were no significant differences between type I and type II carcinomas with regard to the $\mathrm{APT}_{\text {mean }}$. Related reports suggest that the tumor APT signal is positively correlated with cell density and proliferative capacity. Furthermore, this is attributed to an increase in the concentration of mobile proteins and peptides in the cell $[15,19,21]$. Hence, high cellularity contributes to increased APT signal. Nuclear atypia is another possible factor related to increased APT signals, owing to interactions with hydrophobic cell membranes and macromolecules [22-24]. Serous and clear cell carcinomas also exhibited higher $\mathrm{APT}_{\max }$ signals. They are suggested to be marked nuclear atypia, prominent mitoses, and proliferation of solid components $[8,9]$. Endometrioid carcinoma is classified into architectural grades (grades 1-3), in accordance with the percentage of solid part as defined by FIGO; grades 1 and 2 contain
Fig. 5 Receiver operating characteristic (ROC) curves show the results of the analysis of $\mathrm{APT}_{\max }$ obtained by reader 1 (a) and reader 2 (b). Details of area under the curves (AUC) and $95 \%$ CIs of each index are shown in the "Results" section
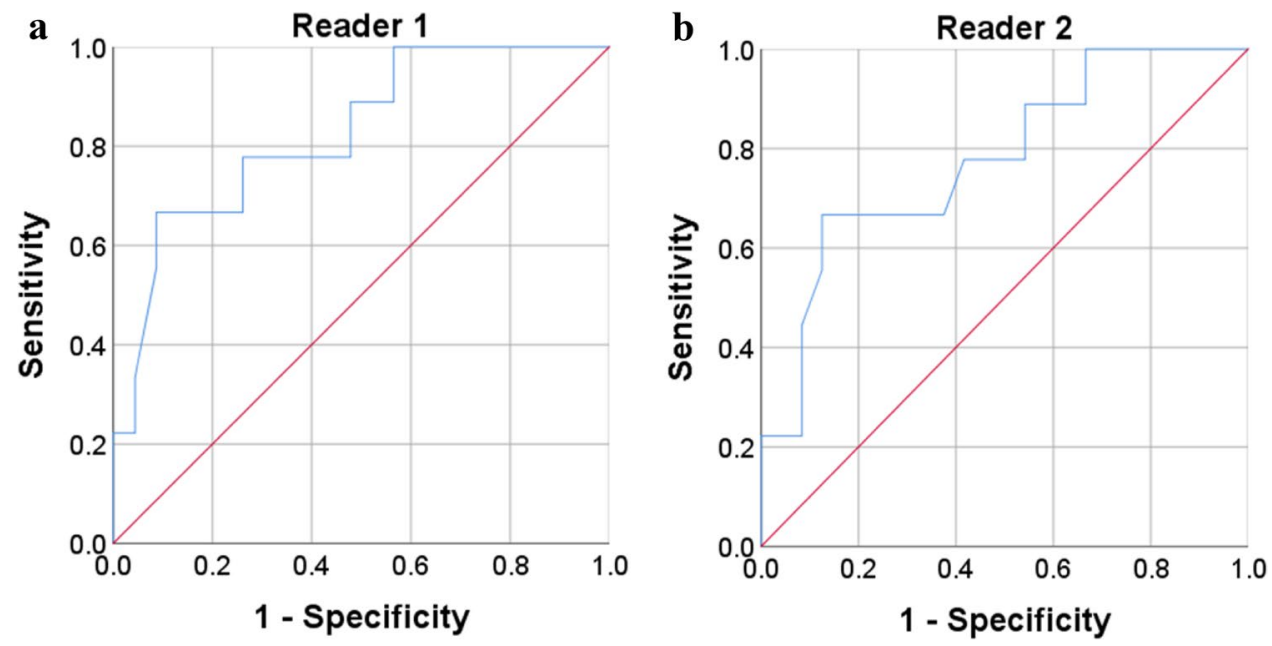
less than $50 \%$ solid part and are predominantly glandular, whereas grade 3 contains more than $50 \%$ solid part as well as marked nuclear atypia $[9,19]$. These differences, including cellularity and nuclear atypia, may contribute to the abundant concentration of mobile proteins and peptides presented by type II carcinomas, namely the higher $\mathrm{APT}_{\max }$ signal. Nevertheless, in the present study, the APT signal was not correlated with the ADC value, reflecting tumor cellularity. Similarly, $\mathrm{APT}_{\text {mean }}$ did not show a certain increase according to the tumor grade in endometrioid carcinoma. These results may be due to the small number of cases investigated. Moreover, other factors, in addition to nuclear atypia and prominent mitoses, may have contributed to the APT signal much more.

In general, $\mathrm{APT}_{\max }$ can indicate the area with the highest local cell density or the most active metabolic activity. High APT signal spots were found in the border area between the tumor and the myometrium, particularly in type II carcinoma. According to a recent study by Gatius et al. [25], the proteomic and metabolic features differ between peripheral and inner cells in tumors with myometrial invasion. In general, type II carcinomas tend to be invasive in nature compared to type I carcinomas. This difference may produce high APT signal spots in type II carcinomas, which reflect active metabolism at the intra-myometrial tumor front. Furthermore, necrosis can increase the APT signal [15, 20]. Although we drew the ROI excluding necrosis, microscopic necrosis could exist in the ROI. Additionally, morphological differences, including cytological (columnar, mucinous, or squamous) or architectural (glandular, papillary, or solid) features, are frequently observed in the same tumor [25]. Thus, we believe that tumor heterogeneity can affect the results. Therefore, APT may be a promising imaging marker that provides a good representation of not only the histopathological characteristics but also tumor heterogeneity related to the microenvironmental metabolic characteristics of the tumor.

The present study included grade 3 endometrioid carcinoma as type II, and it is debatable whether grade 3 endometrioid carcinoma is type I or type II. However, Voss et al. [26] reported that grade 3 endometrioid carcinoma may be more suitable for inclusion in the category of type II, as the immunohistochemistry and survival profiles are similar to those of serous and clear cell carcinomas. Moreover, the conventional classification of endometrial carcinoma into type I and type II has been recently questioned, owing to the frequent disagreement between pathologists with regard to the diagnosis due to the similarity of the histological characteristics pertaining to grade 3 endometrioid and serous carcinomas [8-11].

Previous studies have also attempted to differentiate type II carcinoma from type I carcinoma using MRI.
Regarding ADC values, a recent study showed that the mean and minimum ADC values were significantly lower in type II carcinoma than in type I carcinoma [27], although we did not obtain the same results. Differentiation was assessed using dynamic contrast-enhanced MRI [12] and MR spectroscopy [28]. However, these evaluations are debatable. $\mathrm{APT}_{\max }$ can show the tumor microenvironment, as mentioned above. Therefore, it may provide more detailed information that would not be available with conventional evaluation methods.

The current study has several limitations. First, the number of cases was relatively small, especially in cases of type II carcinoma. Second, APT imaging was performed for only one section per patient, owing to the time limitations of the imaging protocol. To visualize all areas of a tumor, multiple scans, such as the 3D protocol, are suitable and should be employed in future studies involving the same. Third, we excluded four cases because of inappropriate slice selection. APT imaging is performed before the administration of contrast media, as contrast media affects CEST contrast [29]. In these cases, we were not able to obtain the image with the largest tumor area because the post-contrast enhanced images showed the outline of the tumor more clearly than pre-contrast enhanced images. Finally, we believe that we cannot completely exclude the impact of artifacts, such as those caused by intestinal motion, on the APT signal, in relatively small lesions, although APT imaging was performed using the SSFSEbased sequence. In particular, the relatively long examination time for APT imaging is a major problem as artifacts caused by intestinal motion are strongly affected. To solve this problem, we have to shorten the TR using various methods. For example, using a continuous wave instead of a phase cycle as the RF type would shorten the saturation time or reduce the number of refocus RF pulses of SSFSE.

In conclusion, $\mathrm{APT}_{\max }$ was observed to be higher in type II than in type I endometrial carcinoma. APT imaging has the potential to determine the type of endometrial cancer, which can facilitate preoperative decision making.

Acknowledgements We thank Hiroaki Komatsu for their clinical support in the tumor board, as well as Kanae Nosaka for her assistance with the pathological diagnosis and advices.

Funding This research did not receive any specific grant from funding agencies in the public, commercial, or not-for-profit sectors.

\section{Declarations}

Conflict of interest Coauthors Mitsuharu Miyoshi and Atsushi Nozaki are employees of GE Healthcare.

Ethical statement All applicable institutional and/or national guidelines for the care and use of animals were followed. 
Open Access This article is licensed under a Creative Commons Attribution 4.0 International License, which permits use, sharing, adaptation, distribution and reproduction in any medium or format, as long as you give appropriate credit to the original author(s) and the source, provide a link to the Creative Commons licence, and indicate if changes were made. The images or other third party material in this article are included in the article's Creative Commons licence, unless indicated otherwise in a credit line to the material. If material is not included in the article's Creative Commons licence and your intended use is not permitted by statutory regulation or exceeds the permitted use, you will need to obtain permission directly from the copyright holder. To view a copy of this licence, visit http://creativecommons.org/licenses/by/4.0/.

\section{References}

1. Morice P, Leary A, Creutzberg C, Abu-Rustum N, Darai E. Endometrial cancer. Lancet. 2016;387:1094-108.

2. Arora V, Quinn MA. Endometrial cancer. Best Pract Res Clin Obstet Gynaecol. 2012;26:311-24.

3. Braun MM, Overbeek-Wager EA, Grumbo RJ. Diagnosis and management of endometrial cancer. Am Fam Physician. 2016;93:468-74.

4. Brooks RA, Fleming GF, Lastra RR, Lee NK, Moroney JW, Son $\mathrm{CH}$, et al. Current recommendations and recent progress in endometrial cancer. CA Cancer J Clin. 2019;69:258-79.

5. Prat J. Prognostic parameters of endometrial carcinoma. Hum Pathol. 2004;35:649-62.

6. Beddy P, O'Neill AC, Yamamoto AK, Addley HC, Reinhold C, Sala E. FIGO staging system for endometrial cancer: added benefits of MR imaging. Radiographics. 2012;32:241-54.

7. Bokhman JV. Two pathogenetic types of endometrial carcinoma. Gynecol Oncol. 1983;15:10-7.

8. Goebel EA, Vidal A, Matias-Guiu X, Blake GC. The evolution of endometrial carcinoma classification through application of immunohistochemistry and molecular diagnostics: past, present and future. Virchows Arch. 2018;472:885-96.

9. Bell DW, Ellenson LH. Molecular genetics of endometrial carcinoma. Annu Rev Pathol. 2019;14:339-67.

10. Murali R, Soslow RA, Weigelt B. Classification of endometrial carcinoma: more than two types. Lancet Oncol. 2014;15:e268-78.

11. Remmerie M, Janssens V. Targeted therapies in type II endometrial cancers: too little, but not too late. Int J Mol Sci. 2018;19:2380.

12. Fukunaga T, Fujii S, Inoue C, Kato A, Chikumi J, Kaminou T, et al. Accuracy of semiquantitative dynamic contrast-enhanced MRI for differentiating type II from type I endometrial carcinoma. J Magn Reson Imaging. 2015;41:1662-8.

13. Uterine neoplasms, NCCN Clinical Practice Guidelines in Oncology, version 2. (2020). https://www.nccn.org/professionals/physi cian_gls/pdf/uterine.pdf. Accessed Sept 2020.

14. Kamimura K, Nakajo M, Yoneyama T, Takumi K, Kumagae Y, Fukuhara Y, et al. Amide proton transfer imaging of tumors: theory, clinical applications, pitfalls, and future directions. Jpn J Radiol. 2019;37:109-16.

15. Togao O, Yoshiura T, Keupp J, Hiwatashi A, Yamashita K, Kikuchi K, et al. Amide proton transfer imaging of adult diffuse gliomas: correlation with histopathological grades. Neuro Oncol. 2014;16:441-8.
16. Ohno Y, Yui M, Koyama H, Yoshikawa T, Seki S, Ueno Y, et al. Chemical exchange saturation transfer MR imaging: preliminary results for differentiation of malignant and benign thoracic lesions. Radiology. 2016;279:578-89.

17. Takayama Y, Nishie A, Sugimoto M, Togao O, Asayama Y, Ishigami K, et al. Amide proton transfer (APT) magnetic resonance imaging of prostate cancer: comparison with Gleason scores. MAGMA. 2016;29:671-9.

18. Li L, Chen W, Yan Z, Feng J, Hu S, Liu B, et al. Comparative analysis of amide proton transfer MRI and diffusion-weighted imaging in assessing p53 and Ki-67 expression of rectal adenocarcinoma. J Magn Reson Imaging. 2020;52:1487-96.

19. Takayama Y, Nishie A, Togao O, Asayama Y, Ishigami K, Ushijima Y, et al. Amide proton transfer MR imaging of endometrioid endometrial adenocarcinoma: association with histologic grade. Radiology. 2018;286:909-17.

20. Li B, Sun H, Zhang S, Wang X, Guo Q. The utility of APT and IVIM in the diagnosis and differentiation of squamous cell carcinoma of the cervix: a pilot study. Magn Reson Imaging. 2019;63:105-13.

21. Zhou J, Blakeley JO, Hua J, Kim M, Laterra J, Pomper MG, et al. Practical data acquisition method for human brain tumor amide proton transfer (APT) imaging. Magn Reson Med. 2008;60:842-9.

22. Meng N, Wang J, Sun J, Liu W, Wang X, Yan M, et al. Using amide proton transfer to identify cervical squamous carcinoma/ adenocarcinoma and evaluate its differentiation grade. Magn Reson Imaging. 2019;61:9-15.

23. Tang Y, Dundamadappa SK, Thangasamy S, et al. Correlation of apparent diffusion coefficient with $\mathrm{Ki}-67$ proliferation index in grading meningioma. AJR Am J Roentgenol. 2014;202:1303-8.

24. Whittaker CS, Coady A, Culver L, Rustin G, Padwick M, Padhani AR. Diffusion-weighted MR imaging of female pelvic tumors: a pictorial review. Radiographics. 2009;29:759-74 (discussion 774-758).

25. Gatius S, Cuevas D, Fernández C, Roman-Canal B, Adamoli V, Piulats JM, et al. Tumor heterogeneity in endometrial carcinoma: practical consequences. Pathobiology. 2018;85:35-40.

26. Voss MA, Ganesan R, Ludeman L, McCarthy K, Gornall R, Schaller G, et al. Should grade 3 endometrioid endometrial carcinoma be considered a type 2 cancer-a clinical and pathological evaluation. Gynecol Oncol. 2012;124:15-20.

27. Chen J, Fan W, Gu H, Wang Y, Liu Y, Chen X, et al. The value of the apparent diffusion coefficient in differentiating type II from type I endometrial carcinoma. Acta Radiol. 2020;29:284185120944913.

28. Zhang J, Cai S, Li C, Sun X, Han X, Yang C, et al. Can magnetic resonance spectroscopy differentiate endometrial cancer? Eur radiol. 2014;24:2552-60.

29. Jones KM, Pollard AC, Pagel MD. Clinical applications of chemical exchange saturation transfer (CEST) MRI. J Magn Reson Imaging. 2018;47:11-27.

Publisher's Note Springer Nature remains neutral with regard to jurisdictional claims in published maps and institutional affiliations. 